\title{
Application of PDE and Mathematical Morphology in the Extraction Validation of the Roads
}

\author{
Fabricio Leonardi, Viviane Sampaio Santiago, Carolina Dias Chaves, Erivaldo Antônio da Silva
}

Department of the Cartography, Faculdade de Ciências e Tecnologia-FCT/UNESP, Presidente Prudente, Brasil.

Email: Leonardibr@gmail.com, vivisamp@gmail.com, carolina.dias.chaves@gmail.com, silva.erivaldo@gmail.com

Received June $13^{\text {th }}$, 2013; revised July $13^{\text {th }}, 2013$; accepted August $10^{\text {th }}, 2013$

Copyright (c) 2013 Fabricio Leonardi et al. This is an open access article distributed under the Creative Commons Attribution License, which permits unrestricted use, distribution, and reproduction in any medium, provided the original work is properly cited.

\begin{abstract}
The digital images generated by remote sensors often contain noises that are inherent in the process of imaging and transmission. The application of digital processing techniques greatly enhances the ability to extract information on surface targets from remote sensing data. When digital images are used with high spatial resolution, one of the problems emerging the high variability of targets presents in such images. From the computational point of view, the use of partial differential equations is favored by the large number of numerical methods showed in the literature. Many of the models are considered non-complex both from the mathematical and computational standpoints, due to the characteristics of explicit equations. This work uses techniques of the partial differential equations (PDE) and mathematical morphology to extract cartographic features in digital images of the remote sensing. The selected study area corresponds to an image containing part of the Mário Covas Ring Road, located in the metropolitan region of São Paulo (SP), Brazil. The results are promising and show the high potential of using mathematical morphology in the field of cartography.
\end{abstract}

Keywords: Extraction Roads; Partial Differential Equations; Mathematical Morphology

\section{Introduction}

With the intense modifications that are occurring in the cities, highways and the agricultural zone, the cartographic update appears as the basic element for the mapping of the perimeters urban and agricultural as well as of the road mesh.

In this direction, it is essential to search a way for the accomplishment of these updates, so that the same ones occur in the objective and satisfactory form. The extraction of highways using images of remote sensing has been developed in some works in the area of picture processing, contributing to the management of traffic and planning of urban and industrial areas.

According to [1], in the area of cartographic sciences, the problem of extraction of features has been of basic importance for over two decades, in the automation of processes of collection of cartographic features, as buildings, rivers, roads, etc.

Being like this, the highways are cartographic features that need constant update, due to its dynamism in function of modifications in its form or texture.

In this work a sub-picture, is acquired through the Quickbird satellite, high resolution spacially.
Due to the high spatial resolution offered by satellite, several applications can be performed, highlighting the mapping of urban and agricultural areas such as registration, transportation, telecommunications and planning.

A problem when working with the high resolution of images is the complexity of its structure, that is, the diversity of targets with different forms, tonalities and textures, such as houses, shades of buildings, automobiles and trees [2].

In this direction, the use of PDE in picture processing of remote sensing, associated with the techniques of mathematical morphology produces satisfactory results in the extraction of roads, since that they are used in models of PDE and by appropriate morphologic operators.

The model of PDE used in the process of smoothing of the image in this work was developed by [3]. This model, part of an only structure, was formulated from the model of Malik and Perona. Although simple, the model of Malik and Perona is of great importance, the main idea suggests a smoothing selection of the image, where the diffusion is more intense in homogeneous regions and less in regions of edges.

Through substitutions and additions of terms, the model of Barcelos and Chen explores the structures of PDEs 
getting superior results.

As the implementations of the model, how much the morphologic routine they had been carried through in software Matlab 7.0?

The objective of this study was to perform the smoothing of the digital image through PDE, and thereafter extracting the highway through suitable morphological operators, for the purpose of cartographic updating.

\section{Partial Differential Equations}

The use of partial differential equations in picture processing has grown significantly in recent years. The basic idea is to modify one given image, curve or surface with a PDE and obtain the expected results as the solution of this equation.

In other words, the use of EDP in picture processing, not only makes possible the use of good computational algorithms as well as, the use of important theoretical results as existence and unicity of solution.

Thus, some researchers had started to develop models with the purpose to remove, or at least, to minimize the noises gifts in digital images. The idea is that this process purifies the images and keeps their main characteristics such as texture and preservation of edges.

The PDE model used in this study was to [3], which will be described in item 4 .

\section{Optimal Smoothing Filter}

There are two physical considerations to determine the appropriate smoothing filter. The first is that the purpose for filtering the image, is to reduce the ranges of scales in which changes in intensity occur. The goal of filtering an image is to reduce the intensity differences. Thus, the result of filtering should be a smooth image. This condition characterizes a low-pass filter, ie a filter that allows passage of only low frequency signals. This condition can be expressed by imposing that the variance is small.

The second consideration is best expressed as a restriction of the spatial domain which we call the restriction of spatial location, each point of the filtered image should be generated from points near and not random point of the image, because the factors that influence the intensity changes are spatially localized. Factors influencing the intensity changes in an image are:

- lighting, which include shadows, light sources and illumination;

- orientation or distance from the observer to the visible surfaces;
- reflective surfaces.

Thus, in their own scales, these factors can be considered as spatially located. Consequently, each point of the filtered image should be generated from an average score close to that, rather than any other medium of random dots.

\section{The Model of Barcelos and Chen}

The model proposed by Barcelos and Chen, deals with smoothing in digital images and the removal of noise. However, there is great concern about the targeting. So it is a model that acts selectively seeking to maintain the contour of the clearest picture possible.

The model Barcelos and Chen is obtained through the equation (see Equation (1)):

with

$$
u(x, y, 0)=I(x, y)
$$

is

$$
\left.\frac{\partial u}{\partial n}\right|_{\partial R}=0
$$

where $\alpha$ is the function that controls the speed of the diffusion process and $\beta$ is a parameter. It is hoped that the large smoothing homogeneous regions suffer and that the boundary regions are preserved. A good choice for $\alpha$ is the function $\mathrm{g}$ is given by:

$$
\alpha=g\left(\left|\nabla G_{\sigma} * u\right|\right)=\frac{1}{1+k\left|\nabla G_{\sigma} * u\right|^{2}}
$$

Every term of the equation is assigned a specific function, such as decrease the process of smoothing near the boundary regions, which produces remarkable effects in edge detection.

\section{Mathematical Morphology}

[4] defines as a mathematical morphology theory to the analysis of spatial structures. It is called morphology because it is the analysis of form and structure of objects; and mathematics in the sense that the analysis is based on set theory, integral geometry and boolean algebra.

[5] cite that mathematical morphology is considered a powerful tool for image analysis, particularly for those applications where geometric aspects are relevant. The authors also mention that the main idea of mathematical morphology to analyze the shape of objects through a geometric model called structuring element.

[6] defines structuring element as a set completely de-

$$
u_{t}=\alpha(x)\left[\alpha(x)|\nabla u| \operatorname{div}\left(\frac{\nabla u}{|\nabla u|}\right)+\nabla \alpha(x) \cdot \nabla u-\beta|\nabla u|(u-I)\right]
$$


fined and known (form and size), that it is compared, from a transformation to the unknown set of the image. The result of this transformation allows evaluate the unknown set.

To make this assessment, the structuring element moves over the image pixel by pixel, covering the whole scene. In the process of displacement of the structuring element of the image, it performs some transformations in the neighborhood of pixels analyzed. The result of this transformation pixel is assigned to the corresponding new image [7].

\subsection{Morphological Operators Used}

Hdome: Operator hdome removes random noise in the input image $(f)$ through the detection of peaks with higher contrast than the prescribed threshold $(h)$ in the structuring element $\left(b_{c}\right)$ chosen. The Expression (1) demonstrates mathematically the operator.

$$
h-\gamma b_{c} h(f)=\gamma b_{c} f-h(f)
$$

Histeq: Operator histeq enhances the contrast of images by transforming the intensity values in an image, or the values in the colormap of an indexed image, so that the histogram of the output image approximately matches a specified histogram.

Binary: The function converts a binary image in shades of gray $(\mathrm{F})$ in a binary image by comparing each pixel to the threshold chosen (A). The values of the pixels below the threshold (A) are all associated with zero (0) and up to a maximum of radiometric resolution of the image. The expression 3 demonstrates mathematically the operator.

$$
X_{k-1}(f)(x)= \begin{cases}1 & \text { if } f(x) \geq k_{1} \\ 0 & \text { othsewise }\end{cases}
$$

Neg: For the morphological operators to act properly on the image, where torque is a necessary condition that the trait of interest has the binary value 1 . Analyzing the result of binarizing can see that the presented result is reversed in relation to the condition mentioned. To correct this reversal is necessary to apply the operator neg.

$$
f^{c}(x)=k_{\min }+k_{\max }-f(x)
$$

Areaopen: Function areaopen removes any component connected to the image $(\mathrm{F})$ with an area less than the threshold (A) limit. The connectivity is given by the structuring element BC. The Expression (5) demonstrates mathematically the operator.

$$
\begin{aligned}
& f \circ(a)_{B_{c}}=\underset{B \in \mathcal{B}_{B_{c}, a}}{\vee} f \circ B \\
& \mathcal{B}_{B_{c}, a}=\left\{X \subset E: X \text { is } B_{C} \text { - connected, Area }(X) \geq a\right\}
\end{aligned}
$$

\subsection{Otsu Method}

For a threshold $t$ we think needs to be done an analysis of the histogram of the image to be ninarized. This process consists in mapping the gray tones of the image to the set $\{0,1\}$, where 0 (zero) denotes the black color and one (1) white color.

The method treats the image histogram as a probability distribution given as follows:

$p k=n k / N$ wherein $n k$ is the number of pixels with gray tone $k, N$ is the total number of pixels, so $p k$ is the probability of a present pixel tone $k$.

Grouping the pixels into two classes $\mathrm{C}_{0}$ and $\mathrm{C}_{1}$ probabilities of each class are given respectively by:

$$
\begin{aligned}
& \omega_{0}=\sum_{k=0}^{t} p_{k} \\
& \omega_{1}=\sum_{k=t+1}^{k \max } p_{k}
\end{aligned}
$$

wherein $k$ max is the largest gray level and, by definition, $\omega_{1}+\omega_{0}=1$.

The mean $\left(\mu_{0}, \mu_{1}\right)$ and variance $\left(\sigma_{0}^{2}, \sigma_{1}^{2}\right)$ classes $\mathrm{C}_{0}$ and $\mathrm{C}_{1}$ calculated as follows:

$$
\begin{aligned}
& \mu_{0}=\sum_{k=0}^{t} k p_{k} / \omega_{0} \\
& \mu_{1}=\sum_{k=t+1}^{k \max } k p_{k} / \omega_{1} \\
& \sigma_{0}^{2}=\sum_{k=0}^{t}\left(k-\mu_{0}\right)^{2} p_{k} / \omega_{0} \\
& \sigma_{1}^{2}=\sum_{k=t+1}^{k \max }\left(k-\mu_{1}\right)^{2} p_{k} / \omega_{1}
\end{aligned}
$$

Then we find the following medias separability between classes:

$$
\begin{aligned}
& \sigma_{W}^{2}=\omega_{0} \sigma_{0}^{2}+\omega_{1} \sigma_{1}^{2} \\
& \sigma_{B}^{2}=\omega_{0} \omega_{1}\left(\mu_{1}-\mu_{0}\right)^{2}
\end{aligned}
$$

where is the variance $\sigma_{W}^{2}$ intraclass and interclass $\sigma_{B}^{2}$ the variances.

Thus we find a $\mathrm{T}$ that maximizes the interclass variance or minimize the intraclass variance. This method tests all possible thresholds and calculates the average scattering for the pixels in each class $C_{0}$ and $C_{1}$.

The method is applied to histogram separated into two peaks, allowing it to be applied to binarizing with a threshold $\mathrm{T}$ which separates the two peaks. In the case of a histogram that shows three peaks applying a single threshold may cause problems, and its mapping to be done as follows: The values of $t_{1}$ (first threshold separating the second peak) and $t_{2}$ (threshold separates the second peak of the third) take the value 1 (one) denoted by white, otherwise get the value 0 (zero), black color.

\section{Technique for Feature Extraction}

To carry out this work we used a two panchromatic subpicture containing an excerpt from Mario Covas Ring 
Road, located in region of São Paulo (SP), Brazil, which was acquired in October 2010, through the Quickbird satellite, high resolution spatial. This image is shown in Figure 1.

According [8] the use of linear filters, although widely used, is not always the most appropriate method of smoothing images, a time that distort the information of borders affecting the identification and distinction of structures of interest in the process of detecting features. In order to reduce losses in regions of edges and smooth homogeneous areas, an alternative is the use of filters nonlinear based on PDEs.

To conduct the study we used Matlab, both for the implementation of the PDE model as for routine morphology. The procedure for the extraction of road is described in Figure 2.

\section{Presentation and Analysis of Results}

The first step was to convert the original image to grayscale, for after that carrying through the smoothing through the PDE model. The result of smoothing by the PDE model of Barcelos and Chen can be seen in Figure 3. The smoothing was performed with a time t equal to 12.

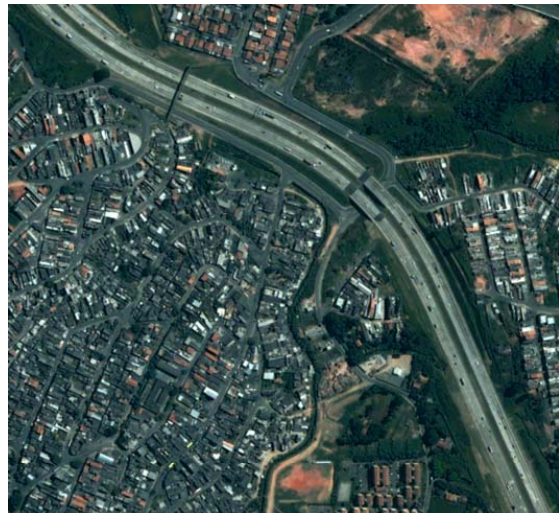

(a)

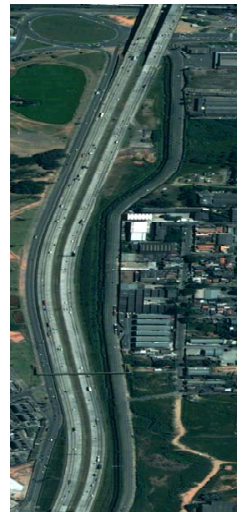

(b)
Figure 1. Original image.

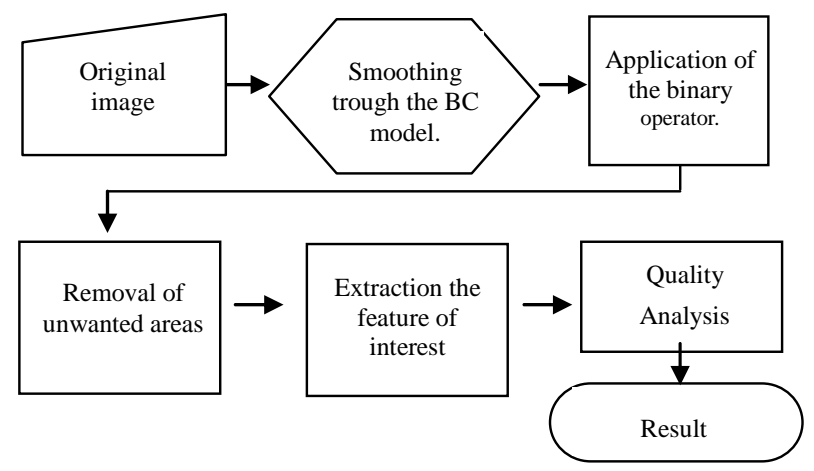

Figure 2. Diagram of algorithm for detecting the feature of interest.
Each of the three terms in the equation has a specific function. The objective of the first term is to smooth both sides of a border region, with conducting a minimum of smoothing the contour. The second term serves to reduce the smoothing process near the boundary and the last term is the approximation of the solution to the original image.

After smoothing the image, it was binarized by Otsu method with threshold 110 and 95, i.e., was transformed into shades of black and white. The values of pixel that they are below of the threshold assume the value 0 (black) and the values that are above assume the value 1 (white).

In order to remove the remaining of area around the highway were applied morphologycal operators. These operators, aims to remove any component with area smaller than a specified value in a binary image.

The Figure 4 shows the results obtained after removal of all targets present in the image that do not correspond to the target of interest highway.

The analysis may be performed visually by overlap-

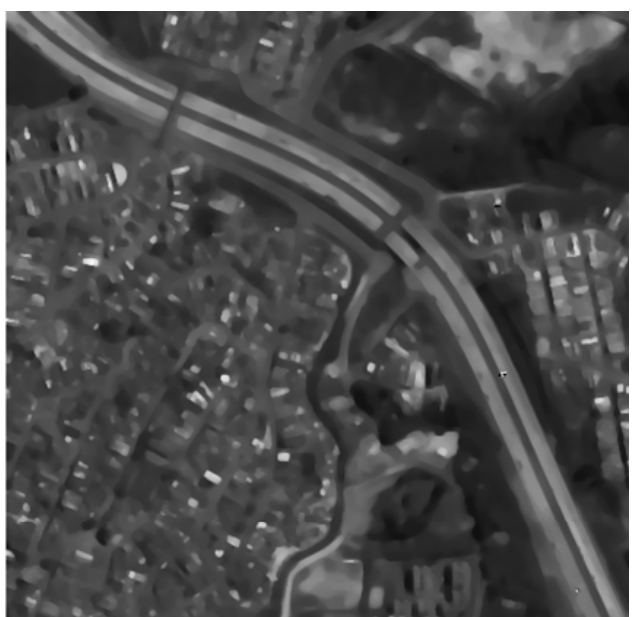

(a)

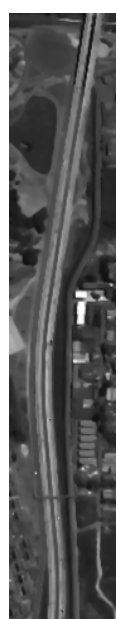

(b)
Figure 3. Result of the application of the Barcelos and Chen model (a) e (b).

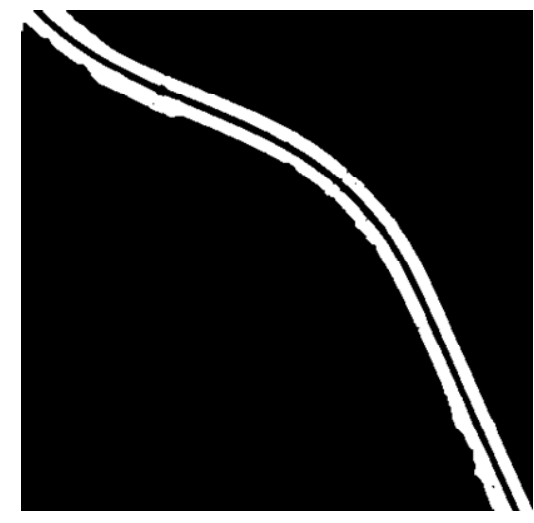

(a)

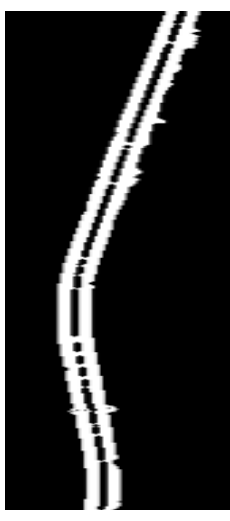

(b)
Figure 4. Result of the extraction (a) e (b). 
ping images, and also numerically, through specific software.

In this study we used the System Analysis of Cartographic Features Extracted for numerical analysis of the results. The results are shown in Figure 5.

To analyze the results are necessary two files, the reference image, in which case is the original image and the end of the extraction process by morphological. The user must select samples of the feature of interest in the original image to be carried out analysis of correspondence between the two images.

It can be considered as a match $(\mathrm{C})$ between the images the value of the ratio between the sum of TB and TB TA and TV as a percentage. The percentage of match can be obtained by the following formula:

$$
C=\left(\frac{T B}{T B+T A+T V}\right) \times 100
$$

where,

- TB represents the total number of white pixels in the image end of the extraction process, which correspond to pixels correctly extracted;

- TV total red pixels, which correspond to pixels not detected in the extraction;

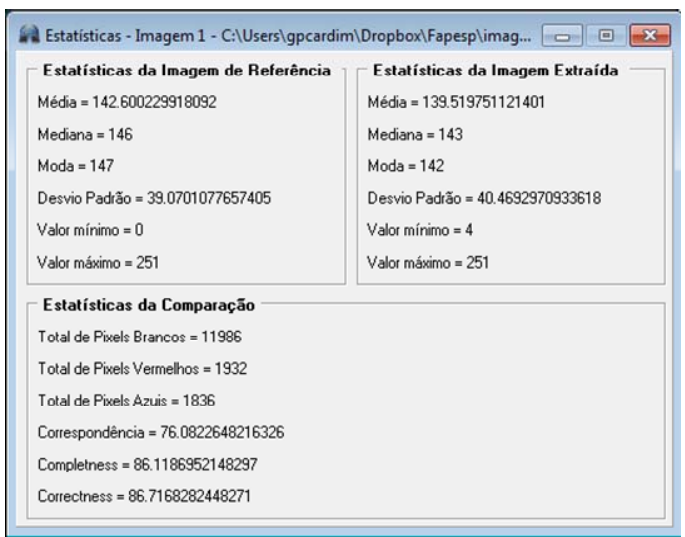

(a)

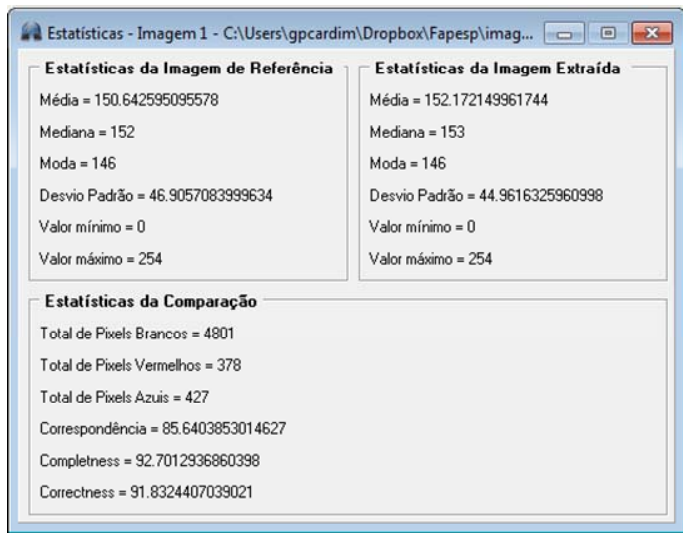

(b)

Figure 5. Result of numerical analysis (a) e (b).
- TA total blue pixels in the image end of the extraction process, which correspond to pixels that are not extracted part of the feature of interest;

By correspondence analysis it was verified that the method is shown suitable for the detection of the feature of interest, with a correspondence of $82.53 \%$. It is noteworthy that the technique of EDP combined with mathematical morphology is effective in extracting of features with features curvilinear.

\section{Conclusions}

From the result it is possible to conclude that the objectives to smooth the image through a PDE model and to carry through the extraction of the features of in- terest with morphologic operators are reached.

The model proposed by Barcelos and Chen had a good result for image smoothing. You can see the edges of the region of interest (in case the roads) are preserved while the image is smoothed. From the computational point of view, the use of partial differential equations is favored due to the characteristic of explicit equations, in addition to the extensive amount of numerical methods in the literature.

The extraction of the feature of interest by routine morphology was also quite satisfactory, resulting in good detection of edges and axes of the tracks.

The choices of thresholds used in the functions must be done carefully, because if they are not suitable, they can cause loss of objects.

The structuring element to be used in functions is the main factor for obtaining good results in detection techniques using Mathematical Morphology, considering that it is the parameter which identifies the shape of the object to be detected.

As the result is satisfactory, the work can be used as a stage of daily preprocessing for processes of automatic extraction of the road mesh, beyond contributing as an alternative method for cartographic update.

\section{REFERENCES}

[1] A. P. Dal Poz, R. B. Zanin and G. M. Vale, "Extração Automática de Feições Rodoviárias em Imagens Digitais,” Revista Controle \& Automação, Campinas, Vol. 18, No. 1, 2007, pp. 44-54.

[2] Y. Chen, B. C. Vemuri and L. Wang, "Image Denoising and Segmentation via Nonlinear Diffusion," Computers Mathematics with Applications, Vol. 39, No. 5-6, 2000, pp. 131-149. doi:10.1016/S0898-1221(00)00050-X

[3] C. A. Z. Barcelos and Y. Chen, "Heat Flow and Related Minimization Problem in Image Restoration,” Computers Mathematics with Applications, Vol. 39, No. 5-6, 2000, pp. 81-97. doi:10.1016/S0898-1221(00)00048-1

[4] P. Soille, "Morphological Image Analysis: Principles and applications,” Springer-Verlag, Berlin, 2003. 
[5] J. Goutsias and H. J. A. M. Heijmans, "Mathematical Morphology,” IOS Press, Amsterdan, 2000.

[6] J. Facon, "Morfologia Matemática: Teorias e Exemplos,” Editora Universitária Champagnat da Pontifícia Universidade Católica do Paraná, Curitiba, 1996.

[7] J. I. Miranda and J. Camargo, "Detecção de Bordas com o Modelo de Difusão Anisotrópica,” Anais XIII Simpósio
Brasileiro de Sensoriamento Remoto, Florianópolis, 2007, pp. 5957-5964.

[8] C. A. Z. Barcelos, "Restauração e Análise de Imagens via Equações Diferenciais Parciais,” Tendências em Matemática Aplicada e Computacional, Vol. 3, No. 2, 2002, pp. 1-13. 